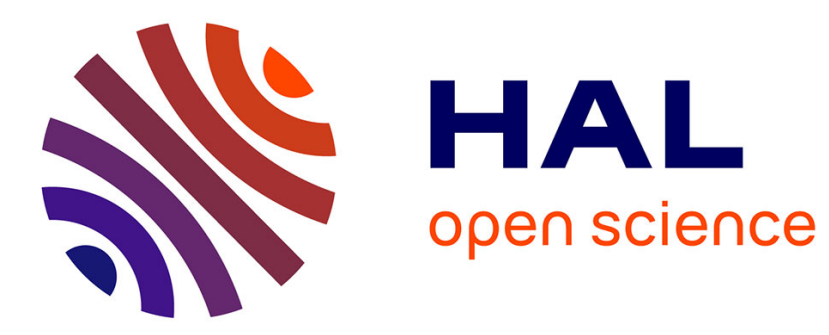

\title{
Nuclear envelope dynamics during plant cell division suggest common mechanisms between kingdoms
}

\author{
Katja Graumann, David E Evans
}

\section{To cite this version:}

Katja Graumann, David E Evans. Nuclear envelope dynamics during plant cell division suggest common mechanisms between kingdoms. Biochemical Journal, 2011, 435 (3), pp.661-667. 10.1042/BJ20101769 . hal-00586467

\section{HAL Id: hal-00586467 https://hal.science/hal-00586467}

Submitted on 16 Apr 2011

HAL is a multi-disciplinary open access archive for the deposit and dissemination of scientific research documents, whether they are published or not. The documents may come from teaching and research institutions in France or abroad, or from public or private research centers.
L'archive ouverte pluridisciplinaire HAL, est destinée au dépôt et à la diffusion de documents scientifiques de niveau recherche, publiés ou non, émanant des établissements d'enseignement et de recherche français ou étrangers, des laboratoires publics ou privés. 


\section{Nuclear envelope dynamics during plant cell division suggest common mechanisms between kingdoms}

Katja Graumann and David E Evans

\section{School of Life Sciences, Oxford Brookes University, Oxford, OX3 0BP, UK}

Corresponding author: David E Evans, School of Life Sciences, Oxford Brookes University, Oxford, OX3 0BP, UK, Tel.: 00441865483968, Fax: 00441865483242, email: deevans@brookes.ac.uk

Short title: Plant SUN domain proteins in cell division

\section{SYNOPSIS}

Behaviour of the NE (nuclear envelope) during open mitosis has been explored extensively in metazoans, but lack of native markers has limited similar investigations in plants. In this study, carried out using living synchronised tobacco BY-2 suspension cultures, the non-functional NE marker LBRGFP and two native, functional NE proteins, AtSUN1 and AtSUN2, we provide evidence that the ERretention theory for NE membranes is applicable in plants. We also observe two apparently unique plant features; location of the NE-membrane components in close proximity to chromatin throughout division and spatially distinct reformation of the NE commencing at the chromatin surface facing the spindle poles and concluding at the surface facing the cell plate. Mobility of the proteins was investigated in the interphase NE, during NE breakdown and reformation, in the spindle membranes and the cell plate. A role for AtSUN2 in nuclear envelope breakdown is suggested.

Keywords: Mitosis / nuclear envelope / Protein dynamics / SUN domain protein / live imaging 


\section{INTRODUCTION}

The NE (nuclear envelope) is a dual membrane structure comprising the outer and inner nuclear membranes, ONM and INM, perforated by nuclear pores. While this basic structure is present in all eukaryotes, there is significant variation in its properties and behaviour between kingdoms. In particular, lower eukaryotes undergo a 'closed' mitosis in which the envelope remains largely intact, while in higher eukaryotes, including plants and higher metazoans, the NE breaks down and reforms in a controlled manner [1]. In metazoans, phosphorylation of chromatin-binding INM components and lamins, which form a supportive meshwork structure underlying the INM, disrupts binding interactions to chromatin affecting the integrity of the NE structure during NEBD (nuclear envelope breakdown). At the end of division, dephosphorylation allows these interactions to be re-established and for the NE to reform around the decondensing chromatin [1]. Two models haven been proposed as to the fate of the NE membranes and components during open mitosis [2]. The vesiculation model is supported by earlier research using cell free Xenopus egg extract to study NE reformation and suggests that NE membranes and protein components are present as vesicles in the dividing cell, which are recruited to the decondensing chromatin in late anaphase to re-assemble into the NE network. The favoured ER (endoplasmic reticulum) retention model stems from more recent research using living, intact cells and proposes that during NEBD the NE membranes and protein components become part of the mitotic ER network and re-emerge from this network at late anaphase to reform the $\mathrm{NE}$ around decondensing chromatin [1-4]. The molecular mechanisms and components essential to the mitotic dynamics of the NE are well researched in metazoans but remain mainly obscure in plants. This is partly due to a lack of known proteins and their functions involved in these processes. While cyclin, cyclin-dependent kinases and aurora kinases are involved in mitotic progression, their impact on NEBD and NE reformation remains unknown [5,6]. Well-documented chromatin-binding INM proteins such as the LBR (lamin B receptor) or LEM (Lap-Emerin-Man) domain proteins are absent in plants. This is also true for lamins. However, recent research of the dynamics of two lamin-like proteins, NMCP1 (nuclear matrix constituent protein) and NMCP2, suggests that these also disassemble at the beginning of mitosis in Apium graveolens cells but are distributed and reassemble differently [7]. The NMCP1 associates with the mitotic spindle, whereas NMCP2 is present in the mitotic cytoplasm and assembles later at the reforming NE than NMCP1 [7]. Whether NMCP1 and 2 function similarly to lamins, however, remains unknown. The same study suggests that in fixed $A$. graveolens cells dye-stained membranes, including the NE, are present in vesicles, supporting the vesiculation model [7]. On the other hand, studies with the human-derived plant NE marker LBRGFP, showed that NE membranes in living BY -2 cells distributed to mitotic membranes supporting the ER retention model $[8,9]$. The same marker was used previously to show that in HeLa cells the NE membranes migrate into the mitotic ER and re-emerge to form the new NE [3]. The study also found that while the LBR-GFP was strongly immobilised in the interphase NE by binding interactions, it moved relatively freely in the mitotic membranes indicative of unbound, free movement. Until recently a lack of native plant NE proteins ruled out examining the fate and function of NE intrinsic components in mitosis. Here, we address this issue by using the newly characterised plant SUN (Sad1/UNC84) domain proteins [10]. The SUN domain proteins are highly conserved across kingdoms and are a key component of bridging complexes that span the nuclear periplasm to link the nucleoskeleton and chromatin with the cytoskeleton [11-13]. The Arabidopsis thaliana SUN domain proteins AtSUN1 and AtSUN2 were found to be localised at the NE in interphase cells and provide first evidence of a functional nucleo-cytoskeletal bridging complex in plants and a unique opportunity to study NE membrane protein targeting and mobility [10]. A NLS (nuclear localisation signal) was found to be involved in their NE targeting and a C-terminal coiled coil domain required for binding interactions between the two SUN domain proteins. They were found to be characteristic, functional NE components [10] and as such are ideal to study mitotic division.

The aims of this study were to gain insights into the fate of NE membranes and their functional components in plant cell division. To do this we observed the localisation and mobile behaviour of the plant SUN domain proteins in interphase and mitosis together with the non-functional LBR-GFP NE marker. Our findings support the ER retention model and show that, in contrast to metazoans, mitotic membranes remain in close proximity to mitotic chromatin and that NE reformation is spatially 
organised in dividing plant cells. Moreover we observed changes in the mobility of AtSUN2-YFP during mitosis indicating alterations in binding inetactions.

\section{MATERIALS AND METHODS Constructs}

The construction of all AtSUN1 and AtSUN2 based fluorescent protein fusion constructs as well as LBR-GFP was previously published $[8,10]$. The pBIB::H2B-YFP [14] was used as template to make H2B-CFP. Forward primer FH2B (5' ATGGCGAAGGCAGATAAGAAACC 3') and reverse primer RH2B (5' AGAACTCGTAAACTTCGTAACCG 3') were used to amplify the H2B coding sequence and primers FH2BGW (5' GGGGACAAGTTTGTACAAAAAAGCAGGCTTCCCGCCAATGGCGAAGGCAGATAAGAAAC C 3') and RH2BGW (5' GGGGACCACTTTGTACAAGAAAGCTGGGTCAGAACTCGTAAACTTCGTAACCG 3') were used to add gateway flanking recombination sequences. Using gateway cloning technology, the $\mathrm{H} 2 \mathrm{~B}$ coding sequence was first inserted into the entry vector pDONR207 and then moved to the binary expression vector $\mathrm{pK} 7 \mathrm{CWG} 2$ to make $\mathrm{pK} 7 \mathrm{CWG} 2:: \mathrm{H} 2 \mathrm{~B}$ (H2B-CFP). The vector was introduced into Agrobacterium tumefaciens GV3101::pMP90 using heat shock transformation [15].

\section{Stable transformation of BY-2 cells}

Co-cultivation adapted from previously published protocols [16] was used to generate stable transformed BY-2 cell lines. Agrobacteria carrying either pCambia1300::AtSUN1-YFP, pCambia1300::AtSUN2-YFP, pCambia1300::YFP-AtSUN1 $\triangle N$, pCambia1300::YFP-AtSUN1 $\Delta C C$, pCambia1300::YFP-AtSUN2, pVKH18En6::LBR-GFP or pK7CWG2::H2B were used to stably transform BY-2 cells. Wild type BY-2 cells were grown at $25^{\circ} \mathrm{C}$ in the dark with shaking at $130 \mathrm{rpm}$ and subcultured every 7 days [16]. For transformation, 3 day old cells were used. Liquid over-night agrobacteria cultures were centrifuged and washed three times with YEB medium containing $200 \mu \mathrm{M}$ acetosyringone. Bacteria were incubated in a final wash for $1 \mathrm{~h}$ before transformation. Bacteria and BY-2 cells were co-cultivated for $72 \mathrm{~h}$ at $25^{\circ} \mathrm{C}$ in the dark without shaking on solid BY-2 mediumcontaining plates. BY-2 cells were then washed three times with liquid BY-2 medium containing carbenicillin and timentin. Finally, cells were plated out on solid BY-2 medium plates containing carbenicillin, timentin and selection antibiotics. H2B-CFP was selected with kanamycin, the other constructs with hygromycin B. Cells were incubated at $25^{\circ} \mathrm{C}$ in the dark without shaking for approximately 4 weeks until calli had grown. Calli were screened with a Leica MZFLIII stereomicroscope for fluorescence and selected calli were transferred to liquid BY-2 medium including selection antibiotics to establish stable expressing BY-2 suspension cell lines. Stable expressing cell lines were grown and cultured like wild type cell lines [16]. For double transformed cell lines, BY-2 cells were first transformed with one construct and once stable expressing suspension cells were established, these were used for a second transformation with the other construct.

\section{Synchrony of stable transformed BY-2 cells}

Synchrony of BY-2 cells was based on a previously published protocol [17]. 7ml of 7d old cells were transferred to $50 \mathrm{ml}$ fresh medium and aphidicolin was added to a working concentration of $5 \mu \mathrm{g} / \mathrm{ml}$. The cells were incubated for $24 \mathrm{~h}$ at $25^{\circ} \mathrm{C}$ with shaking in the dark. The aphidicolin was washed out using a sintered glass funnel. The cells were washed 10 times with $50 \mathrm{ml} \mathrm{BY-2}$ medium and suspended in $50 \mathrm{ml}$ fresh BY-2 medium after the last wash. Cells were returned to the incubator and sampled for analysis between 4 and $11 \mathrm{~h}$ after. At least two independent synchrony experiments were carried out for each cell line.

\section{Confocal microscopy}

Cells were mounted onto glass microscope slides covered in a thin film of BY-2 medium containing $0.7 \%$ low-melting agarose and sealed with a cover slip. Confocal imaging was carried out as described previously [10]. Acquisition and analysis of FRAP (fluorescence recovery after photobleaching) data was as described previously except that no latrunculin B treatment was used. Approximately 30 FRAP 
experiments were carried out for each data set $[10,18]$. Average values \pm standard deviation (SD) are displayed. For movie acquisition, samples were scanned every 30 s for approximately $45-60 \mathrm{~min}$. The images were converted to movie files using RAD video tools.

\section{RESULTS}

\section{Subcellular localisation of SUN domain proteins in dividing BY-2 cells}

To follow the dynamics of the SUN domain proteins and the NE in living, dividing cells, stable transformed BY-2 cell lines were created that co-expressed either histone H2B fused to the fluorescent protein CFP (H2B-CFP) and AtSUN1-YFP or H2B-CFP and AtSUN2-YFP. Histone H2B-CFP is an A. thaliana chromatin marker [14] and was found to localise to the nucleoplasm labelling chromatin in BY-2 cells (Figure 1 and 2; Supplementary Movies 1-3).

As previously reported, AtSUN1-YFP and AtSUN2-YFP localise to the NE in interphase with low levels of fluorescent protein also being present in the ER in some cells (Figure 1; [10]). As the cells entered mitosis, changes in the distribution of the two SUN domain proteins were already noticeable. In prophase, as condensing chromatin was observed, AtSUN1-YFP and AtSUN2-YFP fluorescence at the NE began to decrease and the fusion proteins to accumulate at opposing sides of the NE, presumably where the spindle poles were forming, as well as in the ER (Figure 1 and Supplementary Movie 3). The SUN-labelled NE started to lose its shape (Supplementary Movie 3) and in prometaphase a structured NE was no longer discernible (Figure 1 and Supplementary Movie 1-3). Instead, the two SUN domain proteins were localised in mitotic ER membranes and accumulated in membranes at the spindle (spindle membranes). In particular, AtSUN1-YFP was observed to be present in tubules and also puncta that surrounded condensing chromatin and were in close proximity to it (Figure 1 and Supplementary Movie 2). AtSUN2-YFP was not observed in puncta but also surrounded chromatin, although AtSUN2-YFP fluorescence appeared diffuse (Figure 1 and Supplementary Movie 3). As condensed chromosomes aligned in the division zone in metaphase, both SUN proteins accumulated in spindle membranes close to the spindle poles (Figure 1; Supplementary Movie 1 and 3). However, some AtSUN1-YFP and AtSUN2-YFP fluorescence was observed in tubules stretching through the division zone with tubule tips appearing in close proximity to the chromosomes (Figure 1). In some cells expressing AtSUN1-YFP, the fusion protein was also found in puncta associated with tubules and spindle membranes similar to the prometaphase puncta. As the sister chromatids became separated and migrated to the spindle poles in anaphase, both AtSUN1-YFP and AtSUN2-YFP were observed accumulating on de-condensing chromatin facing the spindle poles (Figure 1; Supplementary Movie 1 and 3). Some tubules were also seen traversing the division zone and in the transition to telophase both SUN fusion proteins appeared in the forming cell plate (Figure 1; Supplementary Movie 1 and 3). In telophase the NE containing AtSUN1-YFP and AtSUN2-YFP reformed around de-condensing chromatin, with the SUN proteins first present on the side of the chromatin facing the spindle poles, then enveloping the sides of the chromatin and lastly surrounding the chromatin facing the growing cell plate (Figure1 and Supplementary Movie 1). In cytokinesis both AtSUN1-YFP and AtSUN2-YFP were present in the expanding NE and in the cell plate and phragmoplast.

Effects of mutations on subcellular localisation of SUN domain proteins in dividing BY-2 cells Previously, we reported that deleting the N-terminus or coiled coil domain from SUN domain proteins affects their mobile behaviour in the interphase NE caused by disruption of binding interactions [10]. Here we examined the effect of these mutations on the distribution of SUN domain proteins in mitotic division. For this we generated stable transformed BY-2 cell lines co-expressing either H2B-CFP and YFP-AtSUN1 $\Delta \mathrm{N}$, where the N-terminus is deleted, or H2B-CFP and YFP-AtSUN1 $\Delta C C$, where the coiled coil domain is deleted. A third cell line was generated that co-expressed H2B-CFP and YFPAtSUN2. Although this fusion protein is full length and not mutated, the fusion of YFP to the Nterminus affects the functionality of the protein altering its mobility and interactions [10]. In the two AtSUN1 mutant cell lines no general effect on the localisation of the SUN proteins was observed in comparison to the non-mutated AtSUN1-YFP cell line (Supplementary Figure 1). However, no puncta 
were observed in prometaphase and metaphase of YFP-AtSUN1 $\Delta \mathrm{N}$ and YFP-AtSUN1 $\Delta \mathrm{CC}$ expressing cells (Supplementary Figure 1). Interestingly, the N-terminal YFP fusion affected the NE localisation of AtSUN2 in interphase BY-2 cells as only weak and indistinct NE labelling was observed in YFP-AtSUN2 expressing cells (Figure 2). In prophase, however, the fusion protein aggregated specifically at the NE (Figure 2 asterix). The localisation of YFP-AtSUN2 in the remaining phases of the division was similar to that of AtSUN2-YFP (Supplementary Figure 2).

\section{Mobility of AtSUN1-YFP and AtSUN2-YFP in mitotic membranes}

Examining the mobility of proteins in the membrane gives information on the binding interactions of these proteins. Slow mobility indicates strong, anchoring binding interactions whereas high mobility suggests the protein is unbound, moving unhindered. Fluorescence recovery after photobleaching (FRAP) was used to quantify the mobile fraction and half time of AtSUN1-YFP, AtSUN2-YFP and LBR-GFP in the interphase NE (Figure 3A and B) and mitotic membranes (Figure $3 \mathrm{C}$ and D, Supplementary Figure 3) of BY-2 cells (Table I and II, Supplementary Table I). The mobile fraction denotes the percentage of fusion protein found moving and the half time is an indicator of how fast this movement is.

In the $3 \mathrm{~d}$ old interphase NE LBR-GFP was very mobile with a mobile fraction of $85.98 \pm 8.19 \%$ and a half time of $1.31 \pm 0.72 \mathrm{~s}$ (Figure 3B, Supplementary Table I). The mobile fraction and half time of LBR-GFP remained unchanged during NEBD, in spindle membranes, during NE reformation and in the cell plate indicating that LBR-GFP remains unbound and freely mobile in these membrane systems (Supplementary Table I, Supplementary Figure 3).

The AtSUN1-YFP had a mobile fraction of $72.66 \pm 5.89 \%$ and a half time of $9.83 \pm 1.75 \mathrm{~s}$ in the interphase NE indicating that over $70 \%$ of the fusion protein were mobile and less then $30 \%$ immobilised in the membrane (Figure 3B, Table I). The mobile fraction of AtSUN1-YFP appeared to remain similar during mitosis as similar mobile fractions were observed for the protein in the metaphase spindle membrane and the reforming NE. In the cell plate, however, the mobile fraction of AtSUN1-YFP was reduced in comparison to the interphase NE indicating less of the protein was mobile (Figure 3B and Table I). A similar half time as in the interphase NE was observed in the reforming $\mathrm{NE}$ and the cell plate. However, in the metaphase spindle membranes AtSUN1-YFP appeared to move faster as the half time was significantly decreased $(5.10 \pm 0.83 \mathrm{~s}, \mathrm{p}<0.001)$.

AtSUN2-YFP had a mobile fraction of $37.82 \pm 8.39 \%$ and a half time of $6.02 \pm 1.62 \mathrm{~s}$ in the interphase $\mathrm{NE}$ indicating that approximately $37 \%$ of the fusion protein population was mobile and approximately $63 \%$ immobile in the membrane (Figure 3B, Table II). During mitosis, the mobile behaviour of AtSUN2-YFP was found to vary significantly. During NEBD the mobile fraction remained low $(31.79 \pm 15.41 \%)$, similar to that of the interphase NE, but it showed a higher rate of movement as indicated by a lower half time of $3.44 \pm 2.18 \mathrm{~s}$ (Table II). This suggests that while AtSUN2-YFP retains its interphase interactions during prophase NEBD, changes occur as ER retention commences. In metaphase spindle membranes, AtSUN2-YFP had almost twice the mobile fraction $(63.46 \pm 7.97 \%)$ of AtSUN2-YFP in interphase and during NEBD (Table II). It also had a shorter half time, indicating that it is much more mobile in metaphase spindle membranes, suggesting that it no longer is part of binding interactions at the interphase NE and during NEBD. As most of the spindle membranes are no longer surrounding chromatin it may be hypothesised that the interphase/prophase interactions of AtSUN2-YFP include binding of chromatin or other nuclear components. This is reinforced by the finding that the mobile fraction of AtSUN2-YFP decreased in the reforming NE, where it was similar to that of interphase and NEBD (Figure 3D and Table II). The half time of AtSUN2-YFP in the telophase reforming NE was higher than during NEBD and similar to that in interphase. In the cell plate, AtSUN2-YFP had a higher mobile fraction $(53.57 \pm 10.29 \%$, Table II) indicating fewer or different interactions than found at the NE. However, as still almost half of the protein is immobilised in the cell plate this may suggest that it is also associated in protein complexes there and may fulfil a function. 


\section{DISCUSSION}

In higher eukaryotes the NE breaks down in open cell division and considerable interest has been shown in the fate of the NE and its components, particularly in metazoans. While work using a Xenopus egg extract system suggested vesiculation of the membrane, more recently the ER retention model has been favoured. In this, the NE is absorbed into the mitotic ER network and re-assembles from this around de-condensing chromatin at the end of division $[2,4,19]$. Whether the ER retention model holds true for the fate of plant NE membranes is still debated. Using both light and electron microscopy, it has been shown that ER derived mitotic membranes are present in the mitotic spindle of plant cells [20,21]. Previously, the non-functional plant NE marker LBR-GFP was shown to be localised in mitotic membranes in dividing BY-2 cells [8,9] providing evidence for the ER retention model. On the other hand, Kimura and colleagues found that in fixed, dividing A. graveolens cells an ER membrane marker, also present in the NE, marked punctate, vesicular structures suggesting vesiculation [7]. While it is possible that in different plant species the NE and ER assume different morphologies in cell division, it is also known that chemical fixation and permeabilisation required for immunostaining affects membrane morphology. To avoid this, and in order to establish whether the ER retention model was also applicable to plants in addition to metazoans, we used living tobacco BY2 cells co-expressing native NE membrane and chromatin markers fused to fluorescent proteins to study the fate of the NE membranes and the functionality of plant SUN domain proteins during mitotic division.

A GFP construct based on human LBR was previously shown to be a plant NE marker that is correctly targeted to the plant NE but is not functional there $[8,18]$. The two plant proteins AtSUN1 and AtSUN2 used in the study are functional NE intrinsic proteins that belong to the SUN domain family, which is conserved across kingdoms [10]. Yeast and animal SUN domain proteins are essential for several mitotic and meiotic functions including anchorage of centromeres and centrosomes as well as decondensation of chromatin at the end of mitosis [13,22,23]. They are also thought to be one of the first INM proteins recruited to the decondensing chromatin during NE reformation [1]. Here, we studied the localisation and mobility of AtSUN1 and AtSUN2 in mitotic membranes to gain first insights into their functionality during cell division.

Our data provides strong evidence that the ER retention model applies to plants as well as to metazoans. Both SUN domain proteins and the LBR-GFP remain in the NE until prophase, upon NEBD migrate to mitotic ER membranes and accumulate in the reforming NE at the end of division (Figure 1; $[8,9]$ ). This redistribution resembles that of metazoan NE intrinsic proteins. The model is therefore likely to be widely applicable to higher eukaryotes undergoing open cell division.

In contrast to metazoans, however, we observe that membranes containing NE proteins remain in close proximity to chromatin throughout division. This may be a plant-specific feature. In metazoans, the NE proteins evenly distribute throughout the mitotic ER and metaphase chromosomes are devoid of membranes $[2,24]$. Our observations show that both functional SUN domain proteins as well as LBRGFP and mutated SUN domain proteins localise to spindle membranes and to tubules traversing the division zone in metaphase, when most of the spindle membranes accumulate at the spindle poles (Figure 1, Supplementary Figure 1-2, Supplementary Movie 1-3; [9]). The presence of the nonfunctional LBR-GFP and SUN mutants in the tubules as well as the high mobility of the AtSUN1-YFP and AtSUN2-YFP in the spindle membranes suggests that the fusion proteins are not necessarily functional in these tubules. However, the presence of the tubules and membranes in close proximity to chromatin throughout mitosis may suggest functional connections. Immunostaining of dividing tomato cells has previously revealed the presence of the NE specific $\mathrm{Ca}^{2+}$ ATPase LCA in spindle associated membranes and it was suggested these membranes are involved in calcium signalling [6,25]. On the other hand, it could be hypothesised that the tubules remain in close proximity to regions of metaphase chromatin, where protein interactions will later initiate NE reformation. In metazoans, mitotic ER tubules containing INM proteins contact decondensing chromatin in anaphase to initiate NE 
reformation $[2,4]$. Güttinger and colleagues suggest that peripheral and core chromatin areas attract different INM proteins and chromatin decondensation plays an essential part in regulating NE reformation [1]. While the role of chromatin and the regulation of both NEBD and NE reformation remain to be elucidated in plants, our observations suggest a functional relationship between mitotic membranes and chromatin in NE reformation. This is corroborated by the finding that NE reformation is spatially organised. Both AtSUN1-YFP and AtSUN2-YFP first aggregate at the surface of chromatin facing the spindle pole followed by accumulation around the sides and finally localisation of the proteins on chromatin facing the cell plate. This directionality has also not been observed in metazoans $[2,4,26]$ and may be a plant specific feature, commensurate with the suggested presence of specific chromatin domains and suggests a tight spatial regulation of NE reformation. Similar observations were made for NE associated NMCP1 and NMCP2 in A. graveolens cells and found that NMCP1 also first assembles on the chromatin surface facing the spindle in late anaphase and at telophase completely surrounded the decondensing chromatin [7]. It is interesting to note that while AtSUN1-YFP and AtSUN2-YFP aggregated in the reforming NE in late anaphase, LBR-GFP did not [9]. Although it is present in membranes surrounding the decondensing chromatin and in the reforming NE, it does not appear to be strongly accumulated in the reforming NE, as observed for the two SUN proteins (Figure 1; [9]). This suggests that AtSUN1 and AtSUN2 are recruited to the reforming NE by specific interactions with either chromatin or other specifically targeted nuclear components such as NMCP1. The accumulation of LBR-GFP in the fully reformed NE is likely due to import of the protein [18] and may therefore suggest that the NE is transport competent at that stage.

By investigating the mobility of the non-functional LBR-GFP in dividing BY-2 cells, we found that it remains highly mobile throughout division (Supplementary Figure 5, Supplementary Table I) and is not involved in binding interactions. Comparisons with AtSUN1-YFP and AtSUN2-YFP mobility are difficult to make, however, because of the varying expression levels between the three constructs (Supplementary Table II).

Our resulst show that the dynamics of AtSUN2-YFP are affected by its localisation in different mitotic membranes. In the interphase NE, during NEBD and NE reformation AtSUN2-YFP remains tightly anchored to the membranes whereas in the spindle membranes and the cell plate the protein is much more mobile. As in the interphase NE, during NEBD and NE reformation AtSUN2-YFP is in close proximity to chromatin and nucleoplasmic components, it suggests that interactions with these immobilise the protein. A possible role for AtSUN2 in prophase and during NEBD is suggested by the finding that YFP-AtSUN2 accumulates at the NE specifically during prophase. What function this may serve remains to be elucidated. In Dictyostelium, SUN1 is required for centromere-centrosome associations [27] and generally yeast and animal SUN domain proteins have been found to associate with chromatin $[12,13,28]$ so a role in chromatin organisation prior to NEBD could be envisaged for AtSUN2. The low signal of YFP-AtSUN2 at the interphase NE suggests that targeting of the protein is inefficient and previous work has shown that an N-terminal NLS is required for effective NE targeting of the protein [10]. The N-terminal YFP fusion was observed to affect the functionality of the AtSUN2 $\mathrm{N}$-terminus [10] indicating that reduced NE targeting of YFP-AtSUN2 in interphase is due to an impaired NLS. The accumulation of YFP-AtSUN2 in prophase is therefore either due to an NLSindependent targeting mechanism or because of an increased permeability of the NE due to the breakdown of NPCs (nuclear pore complexes). In metazoan cells it has been shown that disassembly of NPCs precedes NEBD so that kinases and other components involved in NEBD can accumulate in the nucleoplasm to cause dissociation of interactions at the nuclear periphery and changes in chromatin organisation $[1,4]$.

Finally, all three NE proteins were localised at the cell plate and in phragmoplast membranes between reforming NE and cell plate. Previously, other plant NE components including Ran, RanGAP, WITs (tryptophan-proline-proline [WPP] interacting tail anchored protein) and WIPs (WPP interacting protein) have been found to localise to the cell plate and a role for the Ran cycle in cytokinesis hypothesised [29]. The presence of LBR-GFP, a non functional protein, in the cell plate [8,9] suggests non specific membrane traffic to be responsible for the presence of NE proteins at the cell plate. 
However, AtSUN2-YFP appears to have a significant immobile fraction at the cell plate (Figure 3B, Table II) indicating interactions and therefore may be functional. It is interesting to observe that so far only in plants has spatial NE reformation from proximal to the spindle to proximal to the cell plate, been observed and it is intriguing to speculate whether this is associated with the presence of NE proteins in the cell plate. Amongst other factors such as kinases, phosphatases, Ran and chromatin remodelling components, the amount of available membrane is known to affect NE reformation [4] and it is possible that the cell plate and phragmoplast form membrane reservoirs that affect the distribution of membranes in the plant cell. Thus the SUN domain proteins might be involved in such regulation.

In conclusion, we have obtained convincing evidence for the ER retention model for NE components in living plant cells by showing for the first time that the functional NE proteins AtSUN1 and AtSUN2 relocate to the mitotic membranes in plant cell division. Furthermore, we have demonstrated that in plants some membranes remain in close proximity to chromatin and that the reformation of the NE around de-condensing chromatin is spatially organised starting proximal to the spindle and concluding proximal to the cell plate. Both these observations appear to be unique to plants. We have also shown that the dynamic properties of AtSUN2-YFP change during mitosis and that the protein may be involved in interactions with nuclear components that render it immobile in interphase and are retained during NEBD and NE reformation. A potential role for AtSUN2 in NEBD is hypothesised owing to its immobility at this stage and its specific accumulation in the prophase NE. In addition, its reduced mobility in the cell plate may indicating a potential functional involvement.

\section{AUTHOR CONTRIBUTION}

The experimental work was carried out by Katja Graumann. Experimental design and writing of the manuscript was done by David Evans and Katja Graumann.

\section{ACKNOWLEDGMENTS}

We would like to thank Anne Kearns from Oxford Brookes University for her help with stable BY-2 transformations and culturing of BY-2 cells. We also would like to show our gratitude for Dr. Dennis Francis from the University of Cardiff for providing wild type BY-2 cell lines and his advice on synchronising BY-2 cells

\section{FUNDING}

This work was supported by a grant from the Leverhulme Trust: grant number F/00382/H.

\section{REFERENCES}

1 Güttinger, S., Laurell, E. and Kutay, U. (2009) Orchestrating nuclear envelope disassembly and reassembly during mitosis. Nat Rev Mol Cell Biol. 10, 178-191

2 Hetzer, M. W. (2010) The nuclear envelope. Cold Spring Harb Perspect Biol. 2

3 Ellenberg, J., Siggia, E. D., Moreira, J. E., Smith, C. L., Presley, J. F., Worman, H. J. and Lippincott-Schwartz, J. (1997) Nuclear Membrane Dynamics and Reassembly in Living Cells: Targeting of an Inner Nuclear Membrane Protein in Interphase and Mitosis. J Cell Biol. 138, 11931206

4 Webster, M., Witkin, K. L. and Cohen-Fix, O. (2009) Sizing up the nucleus: nuclear shape, size and nuclear envelope assembly. J Cell Sci. 122, 1477-1486

5 Brandizzi, F., Irons, S. L. and Evans, D. E. (2004) The plant nuclear envelope: new prospects for a poorly understood structure. New Phytol. 163, 227-246

6 Evans, D. E., Irons, S. L., Graumann, K. and Runions, J. (2009) The Plant Nuclear Envelope. In Functional Organisation of the Plant Nucleus (Meier, I., ed.). p. 20, Springer, Berlin 
7 Kimura, Y., Kuroda, C. and Masuda, K. (2010) Differential nuclear envelope assembly at the end of mitosis in suspension-cultured Apium graveolens cells. Chromosoma. 119, 195-204

8 Irons, S. L., Evans, D. E. and Brandizzi, F. (2003) The first 238 amino acids of the human lamin B receptor are targeted to the nuclear envelope in plants. J Exp Bot. 54, 943-950

9 Evans, D.E., Shvedunova, M. and Graumann, K. (2010) The nuclear envelope in the plant cell cycle; structure, function and regulation. Ann Bot., D.O.I. 10.1093/aob/mcq268

10 Graumann, K., Runions, J. and Evans, D. E. (2010) Characterisation of SUN-domain proteins at the higher plant nuclear envelope. Plant J. 61, 134-144

11 Worman, H. J. and Gundersen, G. G. (2006) Here come the SUNs: a nucleocytoskeletal missing link. Trends Cell Biol. 16, 67-69

12 Razafsky, D. and Hodzic, D. (2009) Bringing KASH under the SUN: the many faces of nucleo-cytoskeletal connections. J Cell Biol. 186, 461-472

13 Starr, D. A. (2009) A nuclear envelope bridge positions nuclei and moves chromosomes. J Cell Sci. 122, 577-586

14 Boisnard-Lorig, C., Colon-Carmona, A., Bauch, M., Hodge, S., Doerner, P., Bancharel, E., Dumas, C., Haseloff, J. and Berger, F. (2001) Dynamic analyses of the expression of HISTONE::YFP fusion protein in $A$. thaliana show that synsytial endosperm is divided in mitotic domains. Plant Cell. 13, 495-509

15 Hadlington, J. L. and Denecke, J. (2001) Transient expression, a tool to address questions in plant cell biology. Oxford University Press, Oxford

16 Brandizzi, F., Irons, S.L., Kearns, A. and Hawes, C. (2003) BY-2 cells: culture and transformation for live cell imaging. In Current Protocols in Cell Biology. pp. 1.7.1-1.7.16.

17 Nagata, T., Nemoto, Y. and Hasezewa, S. (1992) Tobacco BY-2 cell line as the "HeLa" cell line in the cell biology of higher plants. Int. Rev. Cytol. 132, 1-30

18 Graumann, K., Irons, S. L., Runions, J. and Evans, D. E. (2007) Retention and mobility of the mammalian lamin B receptor in the plant nuclear envelope. Biol Cell. 99, 553-562

19 Anderson, D. J., Vargas, J. D., Hsiao, J. P. and Hetzer, M. W. (2009) Recruitment of functionally distinct membrane proteins to chromatin mediates nuclear envelope formation in vivo. $\mathbf{J}$ Cell Biol. 186, 183-191

20 Hawes, C.R., E., J.B. and C., H.J. (1981) Low and High Voltage electron-microscopy of mitosis and cytokinesis in maize roots. Planta. 152, 397-407.

21 Gupton, S.L., Collings, D.A. and Allen, N.S. (2006) Endoplasmic reticulum targeted GFP reveals ER organization in tobacco NT-1 cells during cell division. Plant Physiol Biochem. 44, 95105.

22 Tomita, K. and Cooper, J. P. (2006) The Meiotic Chromosomal Bouquet: SUN Collects Flowers. Cell 125, 19-21

23 Chi, Y. H., Haller, K., Peleponese, J. M. and Jang, K. T. (2007) Histone acetyltransferase hALP and nuclear membrane protein hsSUN1 function in decondensation of mitotic chromosomes. J Biol Chem. 282, 27447-27458

24 Anderson, D. J. and Hetzer, M. W. (2008) Shaping the endoplasmic reticulum into the nuclear envelope. J Cell Sci. 121, 137-142

25 Downie, L., Priddle, J., Hawes, C. and Evans, D. E. (1998) A calcium pump at the higher plant nuclear envelope. Febs Letters. 429, 44-48

26 Ellenberg, J. and Lippincott-Schwartz, J. (1999) Dynamics and mobility of nuclear envelope proteins in interphase and mitotic cells revealed by green fluorescent protein chimeras. Methods. 19, $362-372$

27 Schulz, I., Baumann, O., Samereier, M., Zoglmeier, C. and Graf, R. (2009) Dictyostelium Sun1 is a dynamic membrane protein of both nuclear membranes and required for centrosomal associations with clustered centromeres. Eur J Cell Biol. 88, 621-638

28 Xiong, H., Rivero, F., Euteneuer, U., Mondal, S., Mana-Capelli, S., Larochelle, D., Vogel, A., Gassen, B. and Noegel, A. A. (2008) Dictyostelium Sun-1 connects the centrosome to chromatin and ensures genome stability. Traffic. 9, 708-724

29 Meier, I. and Brkljacic, J. (2009) The nuclear pore and plant development. Curr Opin Plant Biol. 12, 87-95 


\section{FIGURE AND TABLE LEGENDS}

Figure 1 Subcellular localisation of SUN domain proteins in synchronised, dividing BY-2 cells Stable transformed BY-2 cells co-expressing either AtSUN1-YFP (green) and chromatin marker histone H2B-CFP (magenta) or AtSUN2-YFP (green) and H2B-CFP. The cells were synchronised using aphidicolin and living cells imaged by confocal microscopy. The two SUN proteins are present in the NE around chromatin in interphase and prophase. Upon NEBD, they distribute to mitotic ER and spindle membranes including tubules traversing the division zone. As the sister chromatids are separated, AtSUN1-YFP and AtSUN2-YFP accumulate in the reforming NE around chromatin first facing the spindle pole and finally proximal to the cell plate. In cytokinesis both fusion proteins are present in the expanding NE, phragmoplast and cell plate. AtSUN1-YFP is present in puncta in prometaphase (asterix) and both SUN domain proteins are present in tubules in close proximity to chromatin (arrow heads). Scale bar $=10 \mu \mathrm{m}$

\section{Figure 2 Distribution of YFP-AtSUN2 in dividing BY-2 cells}

Stable transformed BY-2 cells co-expressing YFP-AtSUN2 (green) and H2B-CFP. The N-terminal fused YFP has previously been shown to disrupt the functionality of the N-terminus of AtSUN2. Consequently the NE targeting of the protein in interphase was reduced as only weak NE fluorescence was observed. In prophase, however, YFP-AtSUN1 labelling of the NE was stronger (asterix) suggesting the protein was accumulated there. Scale bar $=10 \mu \mathrm{m}$

\section{Figure 3 Photobleaching and recovery of NE proteins}

To investigate the mobile behaviour of AtSUN1-YFP, AtSUN2-YFP and LBR-GFP, a selected area (white circle) of the interphase NE, disintegrating $\mathrm{NE}$ (NEBD), spindle membranes (spindle), reforming NE and cell plate was photobleached and fluorescence recovery measured. The raw data was normalised and plotted to obtain recovery curves. A) Interphase BY-2 cell NE labelled with YFPAtSUN1. B) Recovery curves for AtSUN1-YFP, AtSUN2-YFP and LBR-GFP in interphase NE. Fluorescence of LBR-GFP recovered fastest and to the highest level indicating the protein was the most mobile. C) Recovery curves of AtSUN1-YFP in interphase NE, spindle, reforming NE and cell plate. The fluorescence recovered faster in the spindle and to a lower level in the cell plate D) Recovery curves of AtSUN2-YFP in interphase, NEBD, spindle, reforming NE and cell plate. The protein was most mobile in the spindle membranes. $100 \%$ fluorescence intensity indicates the normalised, average pre-bleach fluorescence of each sample. The values for mobile fraction and half time are listed in tables I, II and supplementary table I.

\section{Table 1 Mobility of AtSUN1-YFP in mitotic membranes}

Quantification of mobility of AtSUN1-YFP in interphase NE, spindle membranes, reforming NE and cell plate. The average mobile fractions and half times are displayed $\pm S D(n=30)$. While the mobile fraction is similar in all four membranes, the protein has a shorter half time, and thus moves faster, in the spindle membranes.

\section{Table 2 Mobility of AtSUN2-YFP in mitotic membranes}

Quantification of AtSUN2-YFP in interphase NE, NEBD, spindle membranes, reforming NE and cell plate. The average mobile fractions and half times are displayed $\pm \mathrm{SD}(\mathrm{n}=30)$. The protein is more mobile in the spindle membranes and cell plate as it has a higher mobile fraction and shorter half time in these. 
Biochemical Journal Immediate Publication. Published on 15 Feb 2011 as manuscript BJ20101769

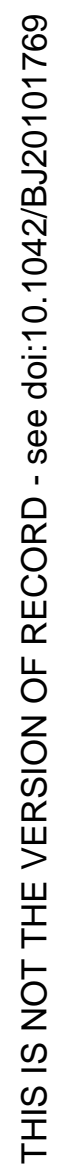

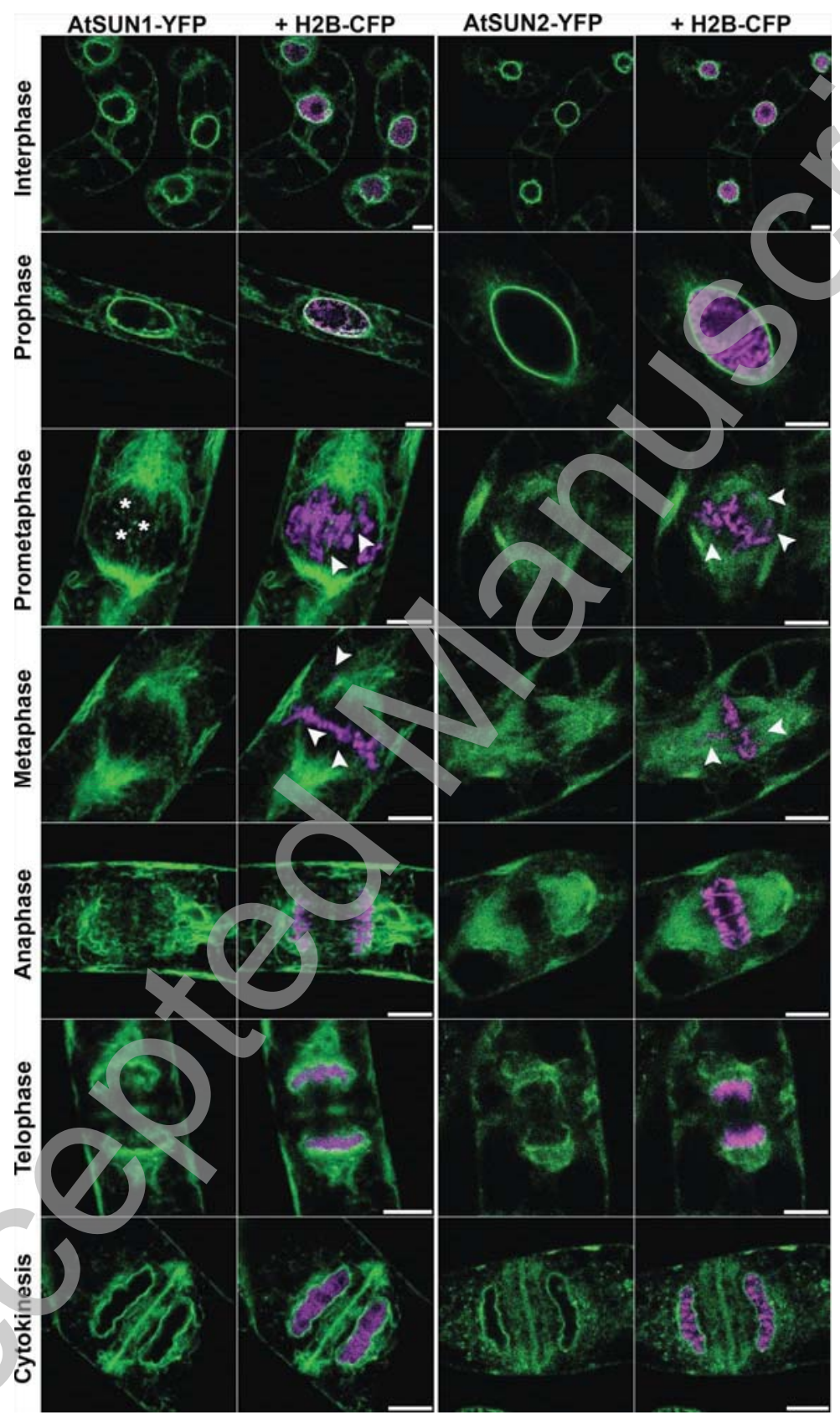

Licenced copy. Copying is not permitted, except with prior permission and as allowed by law. (C) 2011 The Authors Journal compilation (C) 2011 Portland Press Limited 


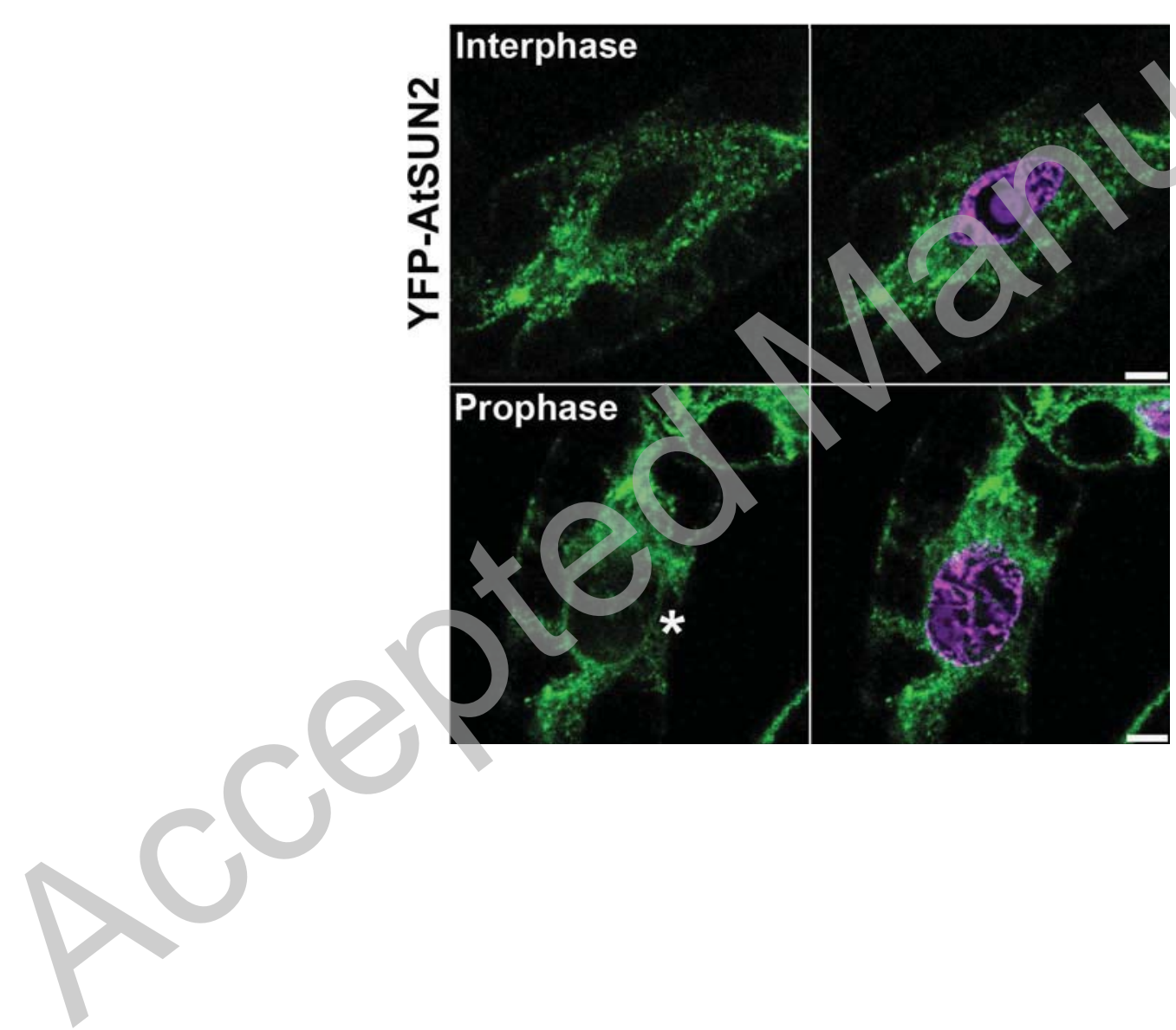

Licenced copy. Copying is not permitted, except with prior permission and as allowed by law. (C) 2011 The Authors Journal compilation (C) 2011 Portland Press Limited 


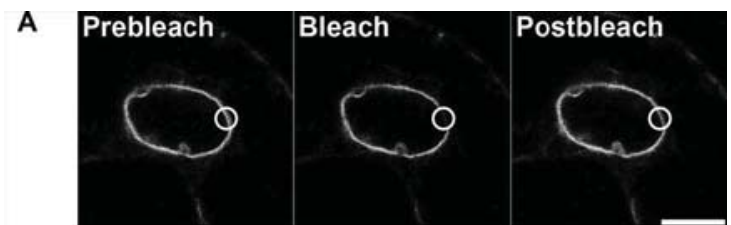

B
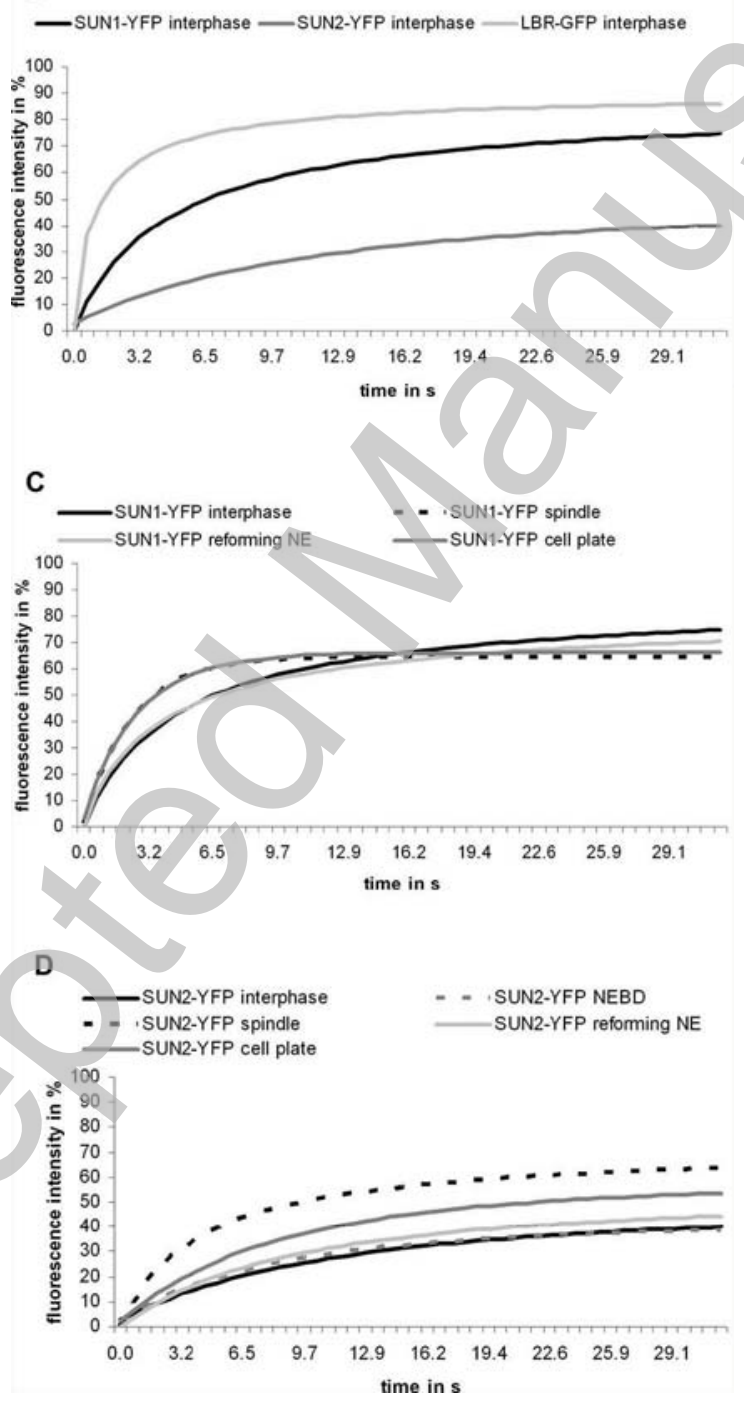

Licenced copy. Copying is not permitted, except with prior permission and as allowed by law. (C) 2011 The Authors Journal compilation (C) 2011 Portland Press Limited 
Table I Mobility of AtSUN1-YFP in mitotic membranes

\begin{tabular}{lcc} 
& Mobile fraction (\%) & Half time (s) \\
\hline 3d Interphase NE & $72.66 \pm 5.89$ & $9.83 \pm 1.75$ \\
\hline Spindle membranes & $68.15 \pm 10.93$ & $5.10 \pm 0.83$ \\
\hline Reforming NE & $69.49 \pm 10.34$ & $8.91 \pm 3.07$ \\
\hline Cell plate & $64.91 \pm 3.87$ & $7.27 \pm 2.46$ \\
\hline
\end{tabular}

Table II Mobility of AtSUN2-YFP in mitotic membranes

\begin{tabular}{lcc} 
& Mobile fraction (\%) & Half time (s) \\
\hline 3d Interphase NE & $37.82 \pm 8.39$ & $6.02 \pm 1.62$ \\
\hline NEBD & $31.79 \pm 15.41$ & $3.44 \pm 2.18$ \\
\hline spindle membranes & $63.46 \pm 7.97$ & $3.11 \pm 0.51$ \\
\hline Reforming NE & $44.33 \pm 12.48$ & $6.55 \pm 3.24$ \\
\hline Cell plate & $53.57 \pm 10.29$ & $5.81 \pm 2.00$
\end{tabular}

